\title{
Identical Burkholderia cepacia complex strain types isolated from multiple patients attending a hospital in Brazil
}

The Burkholderia cepacia complex (BCC) is a group of bacteria comprising at least nine recognized species (or genomovars) (Mahenthiralingam et al., 2005) and associated with various opportunistic human infections. BCC bacteria have been identified in infections of cystic fibrosis (CF) patients and assorted nosocomial infections. Epidemic spread of BCC strains amongst CF patients has been widely documented (Mahenthiralingam et al., 2005). In addition, there have been several reports of outbreaks amongst non-CF patients (Agodi et al., 2002; Souza et al., 2004; Woods et al., 2004; Magalhães et al., 2003; Shehabi et al., 2004). Relatively few studies have identified the BCC species types implicated in an outbreak, and these have generally used PCR-based diagnostic tests targeting the $r e c A$ gene

(Mahenthiralingam et al., 2000). Agodi

et al. (2002) identified episodes of cross-transmission involving Burkholderia cenocepacia and Burkholderia stabilis in non-CF patients. B. cenocepacia has also been implicated in interpatient spread in studies of patients with bacteraemia (Woods et al., 2004), or bacteraemia and respiratory tract colonization (Shehabi et al., 2004). In a previous study of bacteraemia in haemodialysis patients in Recife, Brazil,
B. cenocepacia and Burkholderia vietnamiensis were identified in a polyclonal outbreak (Magalhães et al., 2003). A further study characterized strains as B. cepacia complex or unclassifiable using recA PCR-RFLP analysis (Souza et al., 2004), and water was defined as the source of the outbreak.

Previously, we reported B. cenocepacia as the most prevalent species amongst a collection of non-CF BCC isolates from Brazil, and identified nine B. cenocepacia isolates, from different clinical sources and patients, but sharing a common genotype (Detsika et al., 2003). We also identified isolates from six CF patients that shared the same atypical genotype not corresponding to recognized genomovars (Detsika et al., 2003). Further isolates obtained from in- and outpatients attending the Hospital Portugues in Recife, Brazil, recovered on sheep blood agar or EMB plates, have been identified as belonging to the BCC by using a series of phenotypic tests (Henry et al., 2001). Amongst these isolates are a collection of seven that share phenotypic characteristics (Table 1), with the exception of isolate BC203, which was positive for ornithine decarboxylase. The one CF isolate from this group, BC111 (Table 1), was isolated from one of the six patients in our previous study from whom related isolates of unknown genomovar status were isolated (Detsika et al., 2003). However, isolate $\mathrm{BC} 111$ is different from the previous isolate, which is exemplified by isolate BC14 (Detsika et al., 2003).

The seven isolates could not be assigned to a known species using genomovar-specific recA-based PCR tests, but did yield recA PCR amplicons that were digested with HaeIII (BsuRI) and MspI, as described previously (Mahenthiralingam et al., 2000). The seven isolates shared common recA PCR-RFLP typing patterns with both enzymes (Fig. 1). In order to further confirm their relatedness, we amplified and sequenced a $417 \mathrm{bp}$ region of the outer membrane lipoprotein opcL gene (Plesa et al., 2004) of each isolate for comparison. The opcL sequences of isolates BC92, BC201, BC202, BC203, BC204 and BC205 were identical, and differed by $1 \mathrm{bp}$ from the opcL sequence of isolate BC111. In addition, we sequenced an equivalent $788 \mathrm{bp}$ region of the $\mathrm{rec} A$ genes from strains BC111, BC202, BC203 and BC204. Over this region, the $\operatorname{rec} A$ genes of strains $\mathrm{BC} 202$, BC203 and BC204 were identical, but they differed from the $r e c A$ gene of strain

Table 1. Strains used in this study

Positive $(+)$ or negative $(-)$ results for lysine decarboxylase (Lys), ornithine decarboxylase (Orn), oxidation of sucrose (Suc), oxidation of adonitol (Ado), gelatin liquefaction (Gel), aesculin hydrolysis (Esc), no growth at $42^{\circ} \mathrm{C}\left(42^{\circ} \mathrm{C}\right)$ and $\beta$-haemolysis $(\beta$-h) are shown.

\begin{tabular}{|c|c|c|c|c|c|c|c|c|c|c|c|c|}
\hline Strain & $\begin{array}{l}\text { Isolation date } \\
\text { (month/year) }\end{array}$ & $\begin{array}{l}\text { Patient } \\
\text { age }\end{array}$ & Clinical source & $\begin{array}{l}\text { Inpatient/ } \\
\text { outpatient }\end{array}$ & Lys & Orn & Suc & Ado & Gel & Esc & $42^{\circ} \mathrm{C}$ & $\beta$-h \\
\hline BC92 & $05 / 2002$ & 1 year & Skin (osteomyelitis) & Out & + & - & + & + & + & + & - & - \\
\hline BC202 & $06 / 2003$ & 76 years & Skin wound & In & + & - & + & + & + & + & - & - \\
\hline BC203 & $06 / 2003$ & 20 years & Skin wound & Out & + & + & + & + & + & + & - & - \\
\hline BC111 & $07 / 2003$ & 6 years & CF isolate & Out & + & - & + & + & + & + & - & - \\
\hline
\end{tabular}

${ }^{\star}$ New-born baby may have acquired the infection either as inpatient, from eye-drops following birth, or as outpatient. 


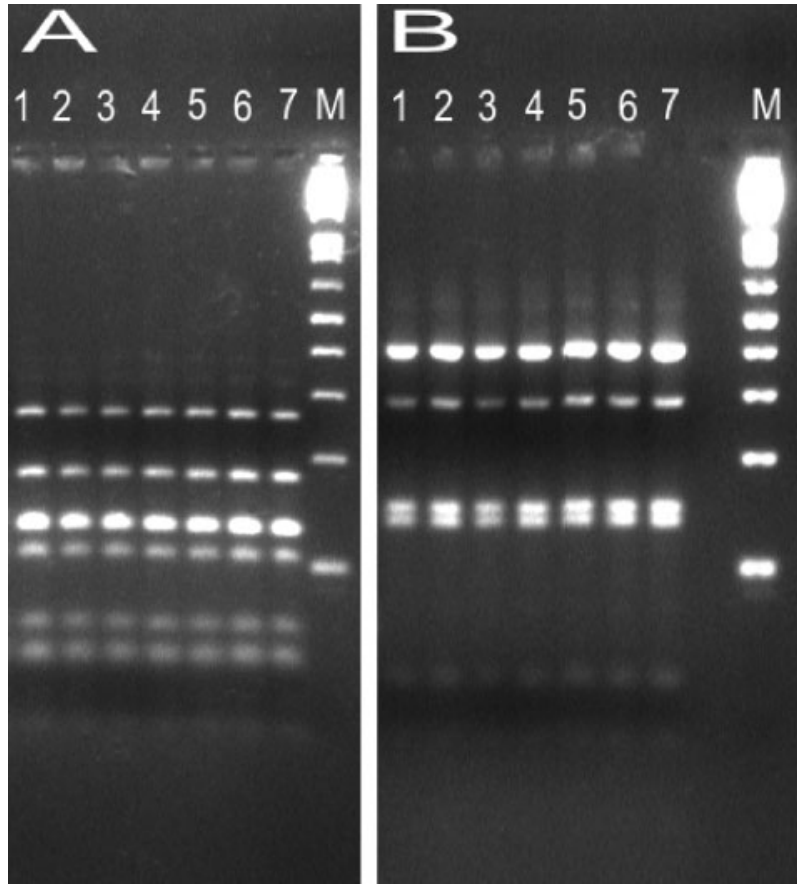

Fig. 1. $r e c A$ PCR-RFLP patterns of new Brazilian isolates. The figure shows $\operatorname{rec} A$ PCR products from the seven Brazilian isolates digested with (A) Mspl or (B) Haelll (BsuRI). The isolates were BC92 (lane 1), BC111 (lane 2) and BC201-BC205 (lanes 3-7). M, $1 \mathrm{~kb}$-Plus ladder (Invitrogen).

BC111 by one nucleotide. The $\operatorname{rec} A$ and opcL sequences have been deposited in
GenBank (accession nos DQ166202 and DQ166203, respectively).
Database searches using the opcL sequences indicated that the best matches with strains of known genomovar status were with $B$. cenocepacia (98\%). The best recA matches were found with isolates of unknown genomovar status (99\%). Sequence analysis indicated that these isolates would not yield amplicons with any of the previously published genomovar-specific primer sets (Mahenthiralingam et al., 2000). The best matches with isolates of known genomovar status were with Burkholderia ambifaria (97\%). Based on the recA sequence, the new isolates (exemplified by BC204) cluster with several isolates of unknown genomovar status and are clearly distinct from the CF isolates of unknown genomovar status reported previously (exemplified by BC14) (Detsika et al., 2003; Fig. 2). The strains HW, R-9929, LMG14095, CEP1061, LMG6860, ATCC17769 and ATCC17460 ( recA sequence accession numbers AF456066, AF456020, AF456016, AF456011, AF456069, AF456008 and AF456019, respectively), which cluster with $\mathrm{BC} 204$ on the basis of $r e c A$ sequence (Fig. 2), are all members of B. cepacia Group K (Vermis et al., 2002; Payne et al., 2005; E. Mahenthiralingam, personal communication). Based on the opcL sequence, the new isolates (exemplified by

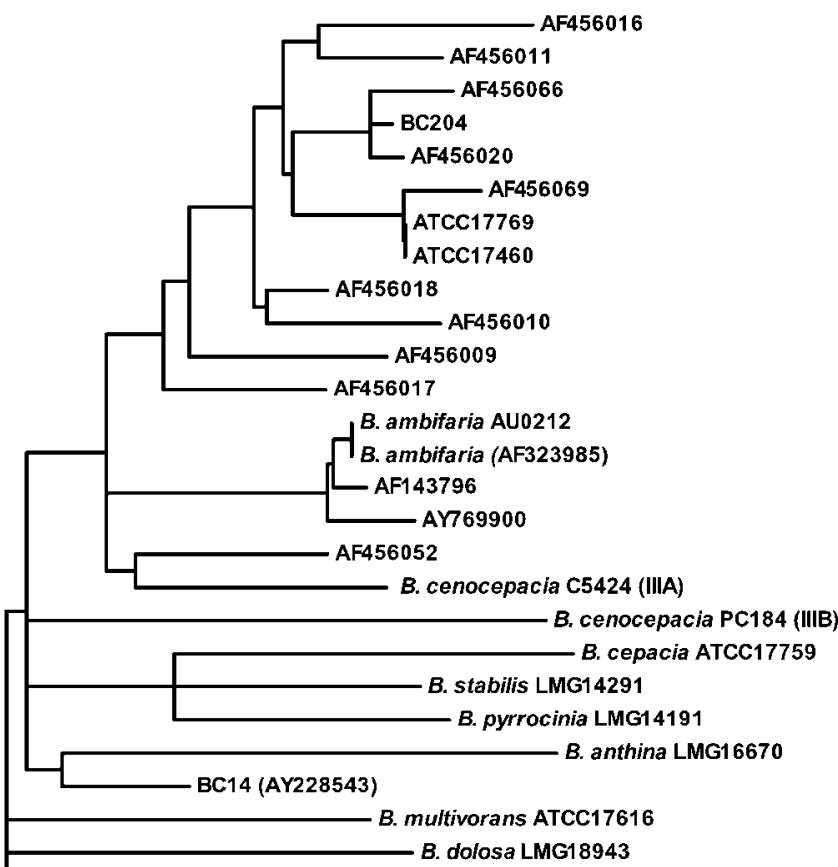

B. vietnamiensis LMG10929
Fig. 2. Phylogenetic relationships according to $r e c A$ sequence comparisons. The dendrogram was constructed using sequences from representatives of known genomovars of the BCC (genomovar I, B. cepacia; II, Burkholderia multivorans; III, B. cenocepacia; IV, B. stabilis; V, B. vietnamiensis; VI, Burkholderia dolosa; VII, B. ambifaria; VIII, Burkholderia anthina; IX, Burkholderia pyrrocinia) and sequences giving best matches in BLASTN searches of the database, as described previously (Winstanley, 2004). $\mathrm{BC204}$ is included as a representative of the new isolates. 


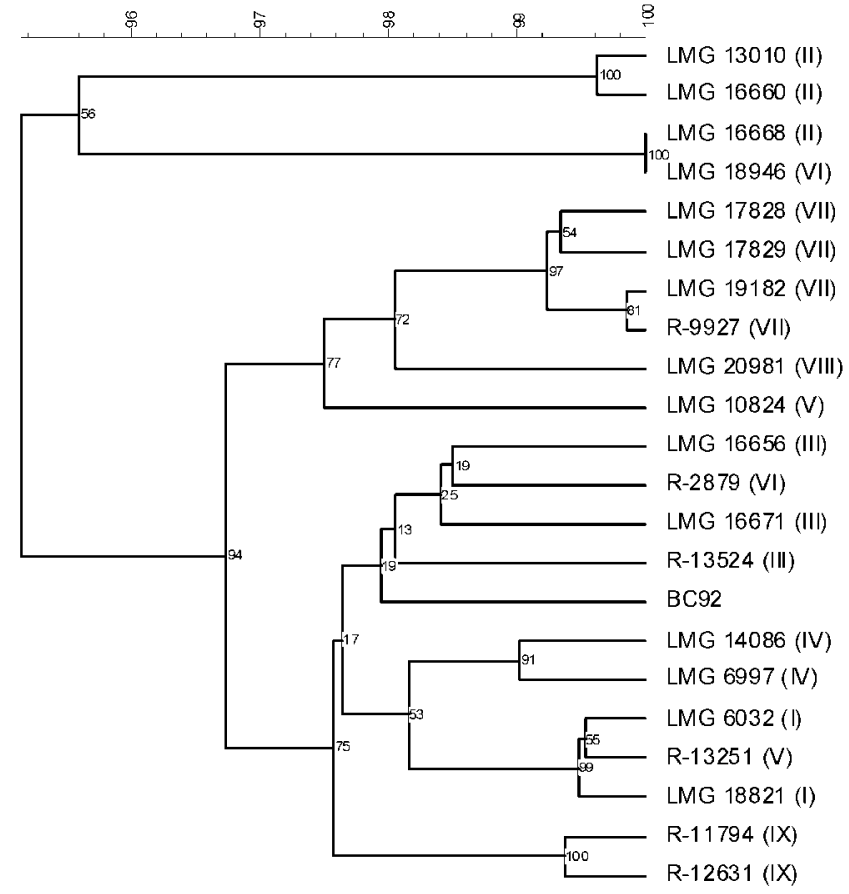

Fig. 3. Phylogenetic relationships according to $o p c L$ sequence comparisons. Sequence alignment and tree construction were carried out as described previously (Plesa et al., 2004). BC92 is included as a representative of the new isolates. Genomovar status is indicated in parentheses (I, B. cepacia; II, B. multivorans; III, B. cenocepacia; IV, B. stabilis; V, B. vietnamiensis; VI, B. dolosa; VII, B. ambifaria; VIII, B. anthina; IX, B. pyrrocinia). Bootstrap values are shown at nodes (based on 100 resamplings).

BC92) clustered most closely with isolates of B. cenocepacia (Fig. 3). However, the nucleotide variations in opcL would lead to one amino acid residue change between the new isolates and $B$. cenocepacia.

Thus, phenotypic and genotypic tests indicate that we have identified a group of related strains isolated from disparate sources and patients. The isolates do not conform to known genomovar status but belong to B. cepacia recA Group K, which may represent another species within the BCC (Payne et al., 2005). The presence of common genotypes amongst such a diverse collection of isolates suggests that this strain type may be common in Brazil.

\section{Wail Assaad, ${ }^{1}$ Marcelo Magalhães, ${ }^{2}$ Maria Plesa, ${ }^{3}$ C. Anthony Hart, ${ }^{1}$ Pierre Cornelis ${ }^{3}$ and Craig Winstanley ${ }^{1}$ \\ ${ }^{1}$ Division of Medical Microbiology and Genitourinary Medicine, University of Liverpool, Daulby Street, Liverpool L69 3GA, United Kingdom}

${ }^{2}$ Department of Tropical Medicine, Federal University of Pernambuco, Recife, Brazil

${ }^{3}$ Laboratory of Microbial Interactions, Department of Molecular and Cellular Interactions, Flanders Interuniversity Institute of Biotechnology, Vrije Universiteit Brussel, Brussels, Belgium

Correspondence: Craig Winstanley (C.Winstanley@liv.ac.uk)

Agodi, A., Barchitta, M., Giannino, V. \& 8 other authors (2002). Burkholderia cepacia complex in cystic fibrosis and non-cystic fibrosis patients: identification of a cluster of epidemic lineages. J Hosp Infect 50, 188-195.

Detsika, M. G., Corkill, J. E., Magalhães, M., Glendinning, K. J., Hart, C. A. \& Winstanley, C. (2003). Molecular typing of, and distribution of genetic markers among, Burkholderia cepacia complex isolates from Brazil. J Clin Microbiol 41, 4148-4153.

Henry, D. A., Mahenthiralingam, E., Vandamme, P., Coenye, T. \& Speert, D. P. (2001). Phenotypic methods for determining genomovar status of the Burkholderia cepacia complex. J Clin Microbiol 39, 1073-1078.

Magalhães, M., Doherty, C., Govan, J. R. \& Vandamme, P. (2003). Polyclonal outbreak of Burkholderia cepacia complex bacteraemia in haemodialysis patients. J Hosp Infect 54, 120-123.

Mahenthiralingam, E., Bischof, J., Byrne, S. K., Radomski, C., Davies, J. E., Av-Gay, Y. \& Vandamme, P. (2000). DNA-based diagnostic approaches for identification of Burkholderia cepacia complex, Burkholderia vietnamiensis, Burkholderia multivorans, Burkholderia stabilis, and Burkholderia cepacia genomovars I and III. J Clin Microbiol 38, 3165-3173.

Mahenthiralingam, E., Urban, T. A. \& Goldberg, J. B. (2005). The multifarious, multireplicon Burkholderia cepacia complex. Nat Rev Microbiol 3, 144-156.

Payne, G. W., Vandamme, P., Morgan, S. H., LiPuma, J. J., Coenye, T., Weightman, A. J., Jones, T. H. \& Mahenthiralingam, E. (2005). Development of a recA gene-based identification approach for the entire Burkholderia genus. Appl Environ Microbiol 71, 3917-3927.

Plesa, M., Kholti, A., Vermis, K., Vandamme, P., Panagea, S., Winstanley, C. \& Cornelis, P. (2004). Conservation of the opcL gene encoding the peptidoglycan-associated outer-membrane lipoprotein among representatives of the Burkholderia cepacia complex. J Med Microbiol 53, 389-398.

Shehabi, A. A., Abu-Al-Soud, W., Mahafzah, A., Khuri-Bulos, N., Abu, K. I., Ouis, I. S. \& Wadstrom, T. (2004). Investigation of Burkholderia cepacia nosocomial outbreak with high fatality in patients suffering from diseases other than cystic fibrosis. Scand J Infect Dis 36, 174-178.

Souza, A. V., Moreira, C. R., Pasternak, J. \& 8 other authors (2004). Characterizing uncommon Burkholderia cepacia complex isolates from an outbreak in a haemodialysis unit. J Med Microbiol 53, 999-1005.

Vermis, K., Coenye, T., Mahenthiralingam, E., Nelis, H. J. \& Vandamme, P. (2002). Evaluation of species-specific recA-based PCR tests for genomovar level identification within the Burkholderia cepacia complex. J Med Microbiol 51, 937-940.

Winstanley, C. (2004). Improved flagellin genotyping in the Burkholderia cepacia complex. FEMS Microbiol Lett 229, 9-14.

Woods, C. W., Bressler, A. M., LiPuma, J. J., Alexander, B. D., Clements, D. A., Weber, D. J., Moore, C. M., Reller, L. B. \& Kaye, K. S. (2004). Virulence associated with outbreak-related strains of Burkholderia cepacia complex among a cohort of patients with bacteremia. Clin Infect Dis 38, 1243-1250. 